\title{
HEAP: A Hierarchical Energy Aware Protocol for Routing and Aggregation in Sensor Networks
}

\author{
Maryam Moazeni and Alireza Vahdatpour \\ Computer Science Department \\ University of California, Los Angeles \\ \{mmoazeni, alireza\}@cs.ucla.edu
}

\begin{abstract}
Applications requiring monitoring such as medical and habitat monitoring, pose a great challenge to sensor network protocols. Power consumption in wireless sensor networks is largely constrained in each node of the network due to the size and the availability of the energy resources. Therefore, having an enhanced scheme that can save transmission energy is ideal. This paper presents an energy efficient and fault tolerant algorithm for routing in wireless sensor networks, which meets sensor networks requirements for monitoring systems that huge amount of events are sensed in them. The algorithm constructs a hierarchy tree over the network and maintains the distribution of energy by restructuring the hierarchy tree over time. The proposed idea was implemented using PARSEC and compared to the HPEQ protocol. Evaluations showed that the proposed algorithm is proper solution for systems with high amount of data sensed because of efficient energy distribution in the network and the ability to aggregate iterative data hierarchically.
\end{abstract}

\section{Categories and Subject Descriptors}

C.2.2 [Computer Communication Networks]: Network Protocols-Routing Protocols; C.2.4 [Computer Communication Networks]: Distributed Systems-Distributed Applications

\section{General Terms}

Design, Performance, Experimentation

\section{Keywords}

Wireless Sensor Networks, Routing, Energy Efficiency

\section{INTRODUCTION}

Wireless sensor networks have become the subject of intense research with recent progress in development of tiny

Permission to make digital or hard copies of all or part of this work for personal or classroom use is granted without fee provided that copies are not made or distributed for profit or commercial advantage and that copies bear this notice and the full citation on the first page. To copy otherwise, to republish, to post on servers or to redistribute to lists, requires prior specific permission and/or a fee. WICON 2007, October 22-24, Austin, USA

Copyright (C) 2007 978-963-9799-12-7

DOI 10.4108/pwsn.2007.2286 processing and sensor systems [2]. One of the most motivating applications of sensor networks is in environmental monitoring systems. Generally, in these systems sensor nodes are distributed in the environment, transmitting data to one or multiple data collection bases (sink nodes), which prepare data for system users or analytical applications. Several characteristics and scenarios can be defined for a monitoring systems. For instance in health care monitoring, the reliability and availability of the system has a high priority. Such systems must guarantee the delivery of event notification messages in a timely manner so that no critical message is missed. On the other hand, in non-critical systems such as weather condition monitoring, increasing the life-time of the system is mostly desirable, while it is endurable to decrease the performance of the network in a reasonable manner.

One of the most challenging issues in designing protocols and algorithms for sensor networks is energy awareness. Due to energy constraints in tiny wireless systems, it is important to evenly distribute the energy consumption across the distributed system in order to increase the total life time of the network. It is possible to address this issue in several layers of the design. In application level this can be done, using dynamic task scheduling algorithms and reconfigurable processing elements which can change their tasks over the time [13].

Furthermore, new hardware solutions such as "mostly off sensors" [15] are utilized in which many of the nodes are kept idle until some events are sensed. However, this technique is not applicable to many scenarios where the generation of events is so frequent which makes it inefficient to apply such technologies. Routing protocols address the communication rate and characteristics in sensor networks which can lead to enormous optimizations in energy dissipation. Basically, existing routing and data aggregation techniques can be divided into three categories [9]:

\subsection{Flat Routing}

In flat routing protocols each node typically plays the same role and sensor nodes collaboratively perform the sensing task. SPIN [5, 10] and Directed Diffusion [8] are of the most well-known data-centric protocols in this category. These two protocols motivated the design of many other protocols which follow similar approaches.

\subsection{Hierarchical Routing}

In hierarchical routing model, a node will be selected as the cluster head to aggregate and route data sensed by other sensor nodes residing in the same cluster. Usually nodes 
with higher energy level are selected for being cluster heads. Employing hierarchical model for aggregating and routing data leads to reduction of the number of transmitted messages among the network nodes and therefore is a proper solution for increasing life-time of the systems [11]. The main advantage of using hierarchical cluster based routing in sensor networks is scalability and communication efficiency. However, most of the work done in this category mainly addresses the problem of data aggregation rather than routing [9].

\subsection{Location-Based Routing}

Location based approach is mainly designed for MANET (Mobile Ad-hoc Network). As opposed to previous routing approaches, in location based approaches geographical location of sensor nodes are used to address them in the network. To acquire the location of nodes, it is possible to communicate to satellites or use GPS (Global Positioning System) or either to obtain the relative location of nodes on the basis of the signal strength passed between neighbor nodes. The common approach for energy saving in this category is to use sleep modes in nodes that are expected to have no activity in a period of time. Due to differences in network constraints, each of the above methods is suitable for special applications. A complete survey of the existing routing protocols can be found in [9].

In this paper we have targeted large scale monitoring systems in which enormous amount of events are sensed (e.g. In-building habitat monitoring) that makes flat routing algorithms inefficient. Flat routing mainly applies flood based data transferring techniques for communication, which will cause high energy consumption in the system and therefore decrease the system life time. Furthermore, location based routing mechanism is not applicable in many cases since additional hardware is required. Additionally, GPS and satellite communication is not available in building structures.

In monitoring systems, adjacent sensor nodes may detect same events and send redundant data to the collection base. To avoid this redundancy that generates unnecessary traffic and dissipates energy inefficiently, a data aggregation method is necessary. However, local aggregation of data which is employed in cluster-based mechanisms is not efficient in many applications. For instance, in tracking applications many sensors can detect the movement of the target and send their sensed event to the collector node while data collected from only a few sensors is enough for this repetitive event. The distribution of iterative sensed data over the large scale network, makes data aggregation using cluster based routing methods in-efficient.

In this paper, we have presented an energy efficient hierarchical multi-hop routing and data aggregation mechanism. This method constructs a hierarchy structure over the network and uses this hierarchy to forward and aggregate data toward the sink nodes. The aggregation mechanism is such that as messages approach the sink node throughout their route, the scope of aggregation becomes wider. Consequently, more messages will be dropped or merged, comparing to previous cluster based methods. The energy efficiency of the method is achieved through evenly distributing the energy consumption among all nodes in routing algorithm. Furthermore, a fast path recovery and restructuring mechanism is embedded in the algorithm in order to overcome the issues and problems forced due to corruption of some nodes or temporary communication errors caused by environmental conditions.

The remainder of the paper is organized as follows: Section 2 discusses some previous related work. Section 3 describes the idea of proposed mechanism and discusses main scenarios such as subscription, path recovery and hierarchy restructuring in detail. Section 4 has experimental results and the discussion about them. Finally in section 5, the conclusion and the future work are presented.

\section{RELATED WORK}

The problem of routing and aggregation in sensor networks has been in the interest recent years. [2], [9], [7], and [14] have done complete surveys on different aspects of the existing routing protocols. The work in this paper is inspired by three well known methods; LEACH [6], HPEQ [8], and Directed Diffusion [8]. LEACH is one of the most cited routing protocols for sensor networks. Basically, it divides the network to some cluster regions and randomly selects a cluster head for each cluster to aggregate data. in this architecture, One hop communication is used to send data from cluster heads to sink nodes.

Directed Diffusion uses multiple routing paths for transferring data to overcome single path failures. Although this method is effective in making the protocol reliable against path failures but its energy consumption and the possibility of collisions and redundant data transfers make it inappropriate for systems with severe energy constraints or high frequency data transfers.

HPEQ is another hierarchical cluster based protocol, which uses LEACH idea for aggregator selection. However, the communication between cluster heads and sink nodes is done using the mechanism introduced in PEQ [3], which uses single paths from source to sink to transfer data packets. HPEQ and LEACH both suffer from the point that they require a lot of message communication between nodes in a cluster for selecting cluster heads. This drawback especially causes harmful effects on the network when the amount of produced data is high and therefore it is needed to change cluster heads periodically to keep the energy consumption distribution of the network balanced.

\section{DESCRIPTION OF HEAP}

The basic idea of HEAP is to construct a hierarchical tree structure over the network. In our scheme, interest messages are used to build the hierarchical structure. The hierarchy is rooted at sink nodes and can be built by assigning a level to each node. Level can be interpreted as the depth of each node in the tree which can potentially grow large and become in the order of network size. Since sensor nodes are constrained with bounded storage, having such a level immediately contradicts the bounded size of storage constraint which was ignored by existing protocols. We observed that nodes do not need to know their exact level since it suffices for each node to be informed of its parent and children level. Therefore, level mod 4 is assigned as the level, which not only limits the level to four numbers but also relative levels of parents and children can be easily determined.

It is possible that a single node belongs to different hierarchical trees and consequently has several levels each associated with one hierarchy. In order to evenly distribute the power consumption over the network, it is reasonable 


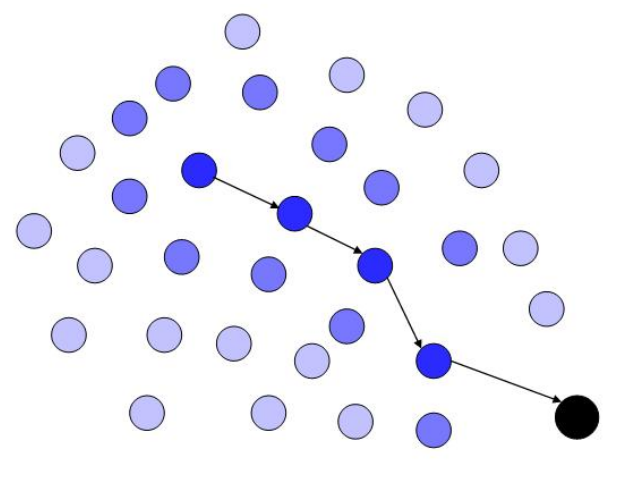

Figure 1: Energy map of a network using referred path

to change the structure of the hierarchy tree over the time. Since changing the structure of the hierarchy tree is costly with respect to message transitions, having an efficient method for restructuring the hierarchy is extremely crucial. In our algorithm, we take advantage of having multiple parents at a time, so that we can implicitly restructure only the portions of the tree that are suffering from the loss of power.

\subsection{Construction of the Hierarchy Structure}

The network needs to be configured before being used to gather data from environment. In the wireless sensor network considered here, one node does not have a global knowledge of the network, i.e. a node only has a small amount of information about its neighbors that are within its wireless coverage range. Therefore, a hierarchy structure is constructed initially. The hierarchy construction is started by sink nodes, which transmit interest messages to their neighbors. A level needs to be associated to each interest message to form the hierarchy. In addition, by each interest message a sink subscribes to one or more nodes by setting one or more criteria.

The algorithm for building the hierarchy is based on broadcasting the interest messages to the network, starting from the sink. Upon receiving the interest message, each node will forward it to neighboring nodes. As mentioned earlier, each node may have several levels and each of them has to be associated with a hierarchy rooted at a certain sink. Moreover, each node has to know the route to the sinks for sending notification messages back to the sinks. Some of the existing protocols [6] use the same path until the path is broken which leads to inefficient energy consumption and extra delay. Figure 1 shows a map that represents the energy consumption in a network when using this method. However, in our scheme each node keeps a list of the nodes that could potentially be used as parents so that one of them is chosen dynamically as the active parent.

The data structure used in the algorithm comprises two tables: hierarchyTable and subscriptionTable. hierarchyTable holds the sinkID, level and parent list. The subscriptionTable is used to store received subscriptions in association with the sinkID. When a node first receives an interest, it regards what the level field denotes as its level and records the transmitter in the parent list along with the sinkID. Since

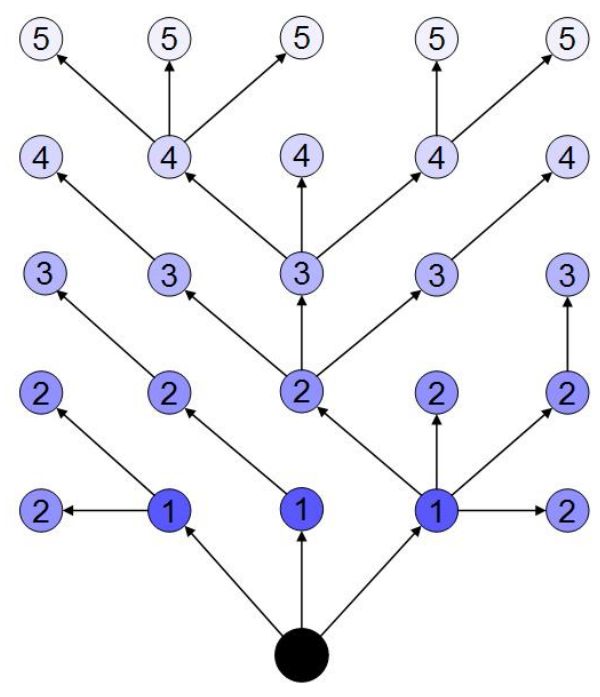

Figure 2: The constructed hierarchical tree structure

then, if another interest message containing the same level is received, the node adds the sender of such message to its parent list. On the other hand, if an arriving interest message contains a larger level than the current level of the node, the message is ignored and will not be forwarded to neighboring nodes. Furthermore, if the interest message contains a smaller level than the current level of the node, the level field in the hierarchyTable is updated and the transmitter is added to the parent list. At the end of the configuration step one of the parents is arbitrary selected from the parent list. Figure 2 shows a hierarchy structure that could be built over a network.

\subsection{Restructuring the Hierarchy Tree}

Restructuring portions of the hierarchical tree that are suffering from the loss of power could favor the network life time. In our algorithm, each parent periodically checks its energy level until some fraction of its energy is consumed and will notify its neighbors to change their parents by broadcasting a CHANGE-PARENT message. Thereafter, the receiving nodes will proceed to the parent changing process. In order to make the energy consumption more distributed, we define a frequency property for each parent in the parent list and the priority is given to parents that has a lower frequency. Figure 3 shows the restructured hierarchical tree over the time, previously shown in figure 2 .

There exist some cases that the nodes are not capable of changing their parents, i.e. they have no other remaining alive parent. Therefore, parent nodes should not wait until they are so close to dead state to notify their neighbors. On the other hand, changing the parent frequently would cause inefficiency by posing additional message transitions. Apparently, deciding on the fraction of energy that should be reached until a node notifies its neighbors is an important factor in keeping the network more stable and robust and will distribute the energy dissipation in the network.

\subsection{Data Aggregation}




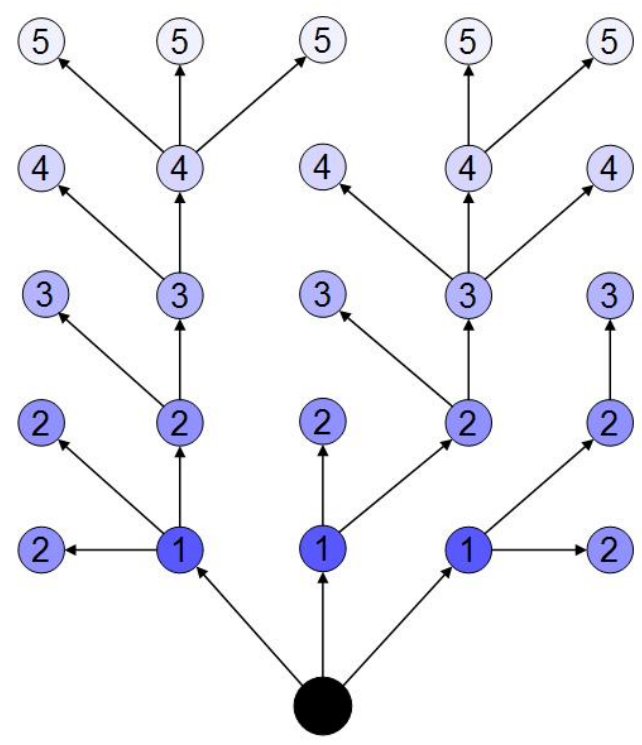

Figure 3: The restructured hierarchical tree structure

Sensor nodes in a certain region may detect the same event and send this redundant data to the sink. To avoid this redundancy that generates unnecessary traffic and dissipates more energy, a data aggregation method is necessary. In our approach for data aggregation, parent nodes in the hierarchy are chosen to be the aggregator which will aggregate the data received from all of children. Therefore, each subset in the network could be seen as a cluster and its root is selected as the aggregator or so called cluster head. Moreover, the process of changing the parent is seen as the expiration of an aggregator and selection of another. It should be noted that there is no message passing involved in the aggregator selection process in HEAP (It is done in conjunction with the parent selection process). Figure 4 shows the clustering of neighboring nodes in a network.

Comparing our aggregator selection mechanism to [6], HEAP will always select the aggregator on a route closer to the sink. In addition, less number of message transitions is made to select the aggregator. Furthermore, In LEACH some nodes may remain out of clusters, which does not occur in HEAP. Moreover, because the aggregators are selected randomly in LEACH they will not necessarily be selected in a proper location relative to other aggregators in the network. In addition, in our approach there is no extra waiting time necessary for the cluster to be set up since each node will independently select its aggregator, which is the same as the selected parent.

\subsection{Path Recovery Mechanism}

Constraints in the energy supply, transient and permanent faults in sensor nodes and the communication blockage could cause failure in the transmission of messages. For overcoming this problem, some existing algorithms apply periodic flooding mechanisms $[8,12]$, rooted at the sink, to repair broken paths and to discover new routes to forward

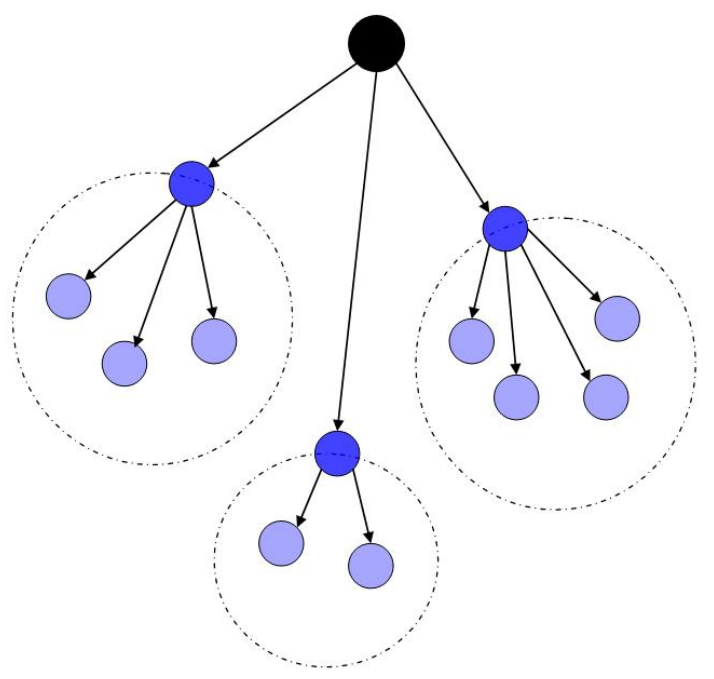

Figure 4: Clustering over the hierarchical tree structure

the traffic around faulty nodes. However, this mechanism is not satisfactory in terms of energy saving because it wastes a lot of energy for flooding the repairing messages. In the path repair mechanism proposed in [4], when the message transmission is failed, the sender will broadcast a SEARCH message and waits for a reply from its neighbors and consequently a new destination is selected by choosing the closest node to the sink. In such mechanisms the transmission of failed messages is delayed until receiving response from neighboring nodes, which can decrease the overall performance of the system especially in noisy environments.

In HEAP recovery scheme when the message transmission fails, the sender needs to mark its active parent as dead and start the parent selection process as described in section 4.3 which makes the path recovery process faster than existing approaches.

\section{SIMULATION EXPERIMENTS}

This section describes the investigation of the performance evaluation of HEAP through an extensive set of simulation experiments. The results are compared to HPEQ routing protocol which was previously compared to Directed Diffusion in [4].

\subsection{Simulation Scenario}

The HEAP algorithm was implemented using PARSEC, a parallel simulation software [1]. Since the arrangement of nodes does not change the results, for the sake of simplicity we chose the mesh to be the arrangement of our network. Table 1 lists the main simulation parameters. To more closely mimic realistic sensor network radios, we utilized an energy model such that the idle time power dissipation was $12.36 \mathrm{~mW}$, the receive power dissipation was $12.50 \mathrm{~mW}$, and the transmit power dissipation was $14.88 \mathrm{~mW}$. Values are based on the values reported for Directed Diffusion in [8, 4]. By average, two events were generated per second.

HEAP and HPEQ were tested with the same simulation scenario and parameters. Each measured value was taken from a mean of 10 simulations outcomes. HEAP is evaluated 
Table 1: Simulation Parameters

\begin{tabular}{|c|c|}
\hline Parameter & Value \\
\hline Simulation time $(\mathrm{s})$ & 1000 \\
\hline Number of nodes & 300 \\
\hline Percentage of source nodes & $2-18$ \\
\hline Source data rate $($ events $/ \mathrm{s})$ & 2 \\
\hline Transmit energy $(\mathrm{mW})$ & 14.88 \\
\hline Receive energy $(\mathrm{mW})$ & 12.50 \\
\hline Dissipation in idle $(\mathrm{mW})$ & 12.36 \\
\hline
\end{tabular}

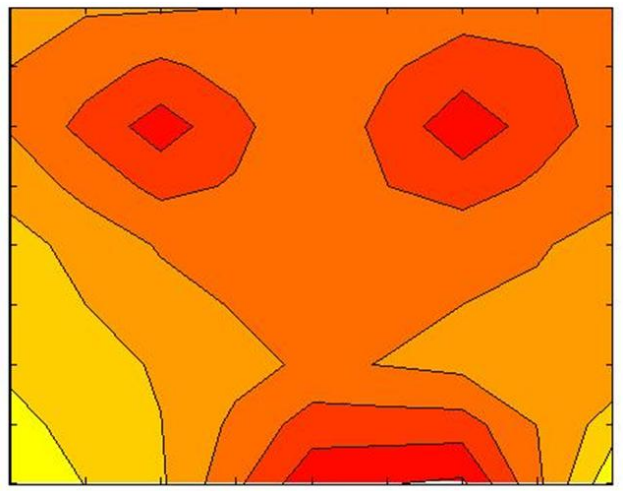

Figure 5: HEAP energy distribution map

through the following metrics:

Average Dissipated Energy The average of the energy dissipated in the all nodes

Average Delivery Ratio The number of received events to the number of sent events ratio

Lifetime of the Network The lifetime of the network after the completion of the configuration step until $50 \%$ of the nodes are shut down

Average Configuration Time The average latency for the complete configuration of the network.

\subsection{Evaluation of HEAP}

HEAP uses a dynamic hierarchical restructuring approach in order to improve the distribution of energy over the network using the aggregator switching procedure. Some of the existing cluster-based protocols $[6,3]$ use a massive message passing scheme for the aggregator switching over time, which is not an efficient approach comparing to the method taken in HEAP where there is considerably fewer message passing involved in the aggregator selection process. Another main drawback of such existing protocols is that the whole network is forced to participate in the process of changing aggregators even though not all regions need to change their aggregator. Therefore, unnecessary message passing will take place to change the structure of existing clusters.

For the purpose of evaluation, a snapshot of nodes energy level in the network was taken after 1000 seconds of the simulation, resulting in the energy map of the network. Figure 5 and 6 illustrates the energy distribution map for HEAP and HPEQ respectively. It can be observed that the energy dissipation is much higher in HPEQ compared to HEAP. The

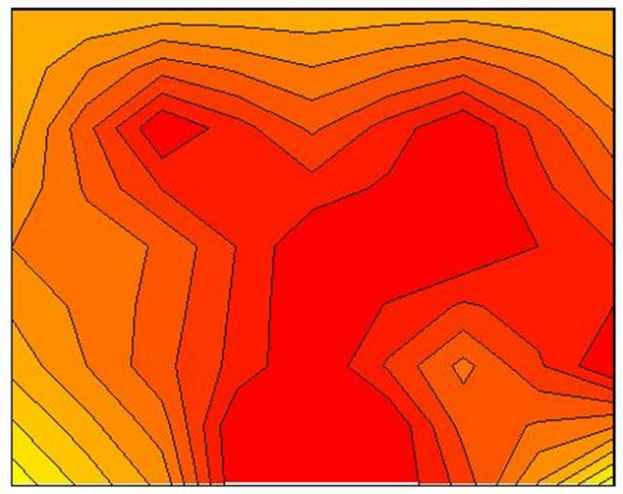

Figure 6: HPEQ energy distribution map

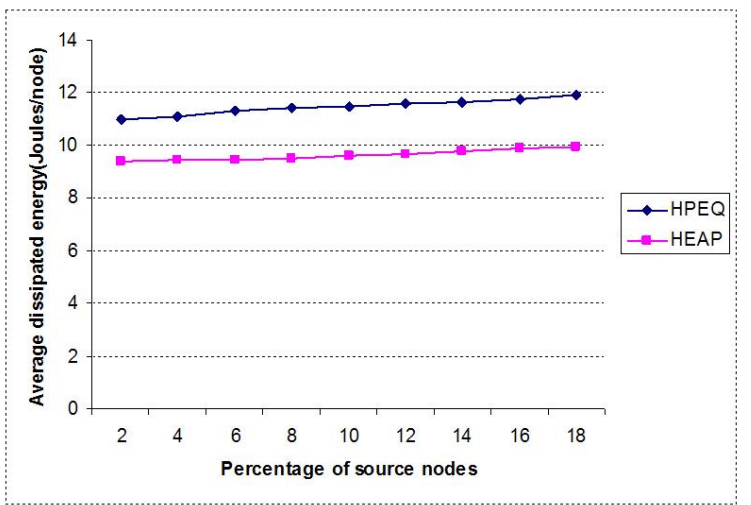

Figure 7: Average dissipated energy

approach taken in dynamic clustering set up (switching of aggregators, clusters configuration) leads to extra overhead to HPEQ. In Figure 6, it can be noted that some regions of the network have rarely been used and energy dissipation is not evenly distributed. However, The HEAP protocol showed a better energy distribution, as seen in Figure 5. The average dissipated energy in nodes surrounding the sink is much higher in HPEQ compared to HEAP. Consequently, the average event delivery ratio is higher in HEAP as illustrated in Figure 7.

Figure 8 depicts that HEAP outperforms HPEQ in terms of average dissipated energy in the network. As mentioned earlier, the message passing approach taken for the aggregator selection and the path recovery in HPEQ results in extra overhead. It can also be noted that in HPEQ, energy dissipation grows with a higher rate by the increase in the percentage of source sensor nodes. Therefore, HEAP maintains a more stable network.

Figure 9 depicts the network life time. It can be observed that HEAP showed better results with respect to the network lifetime. In HEAP, since the energy consumption is more evenly distributed, the number of nodes that shut down decreases. Figure 10 shows the comparison of the average configuration time for two protocols. It can be noted that as the number of sources increases the configuration time spent in HEAP grows in the same order as HPEQ. 


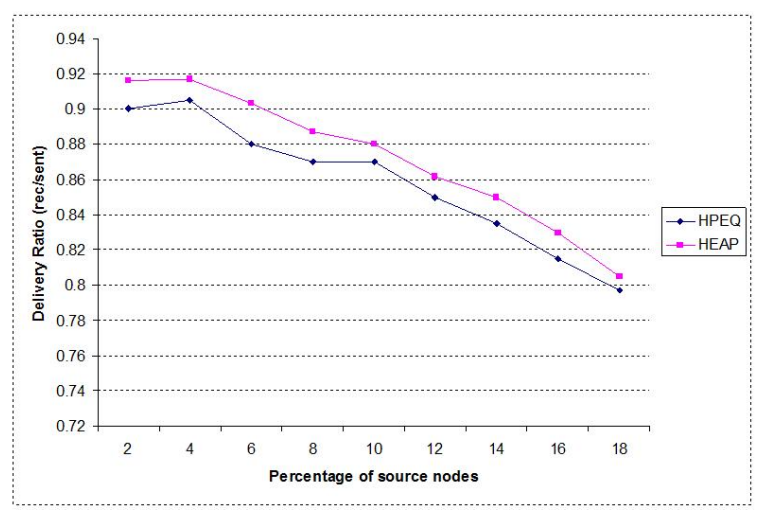

Figure 8: Average delivery ratio

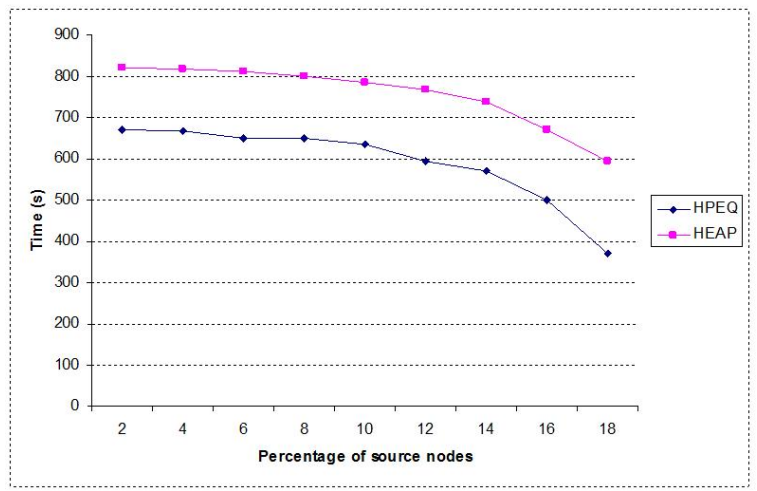

Figure 9: Network life time

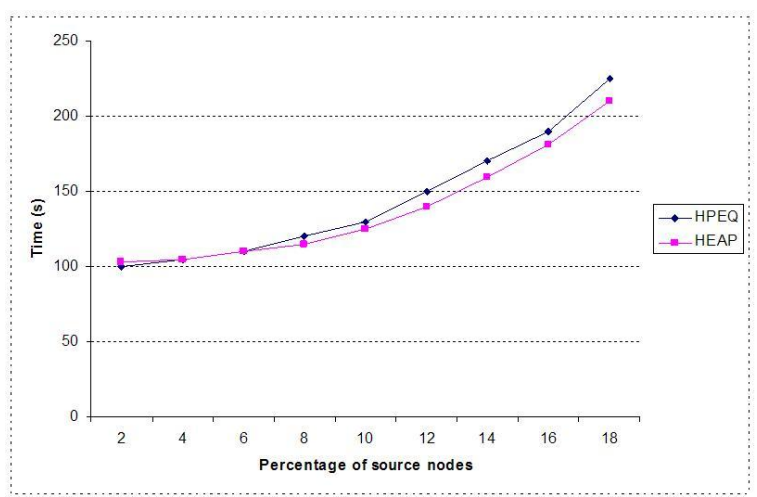

Figure 10: Average configuration time

\section{CONCLUSION AND FUTURE WORK}

A hierarchical aggregation and routing protocol was proposed in this paper. Energy awareness and fast route recovery are the main characteristics of the proposed protocol. It is shown through simulation experiments that the life time of the network is increased about 10comparing to previous well known protocols.

The hierarchical aggregation mechanism embedded in HEAP routing protocol is especially effective in networks with intensive and close sensor nodes. In such networks events are sensed by several neighbor nodes and therefore, iterative data packages are produced at approximately the same time. Therefore, the aggregation can help the network to drop duplicated sensed events and avoid high amount of data transmission in the network.

The effectiveness of HEAP is based on several factors, such as the size of the message buffer, routing and hierarchy level. The performance of the protocol can be optimized through finding the best values for the above factors for different network topologies and sizes.

\section{REFERENCES}

[1] Parsec manual.

[2] K. Akkaya and M. Younis. A survey on routing protocols for wireless sensor networks. Ad Hoc Networks, 3(3):325-349, 2005.

[3] A. Boukerche, R. W. N. Pazzi, and R. B. Araujo. A fast and reliable protocol for wireless sensor networks in critical conditions monitoring applications. In MSWiM '04: Proceedings of the 7th ACM international symposium on Modeling, analysis and simulation of wireless and mobile systems, pages 157-164, New York, NY, USA, 2004. ACM Press.

[4] A. Boukerche, R. W. N. Pazzi, and R. B. Araujo. Hpeq a hierarchical periodic, event-driven and query-based wireless sensor network protocol. In $L C N$ '05: Proceedings of The IEEE Conference on Local Computer Networks 30th Anniversary, pages 560-567, Washington, DC, USA, 2005. IEEE Computer Society.

[5] W. Heinzelman, J. Kulik, and H. Balakrishnan. Adaptive protocols for information dissemination in wireless sensor networks, 1999.

[6] W. R. Heinzelman, A. Chandrakasan, and H. Balakrishnan. Energy-efficient communication protocol for wireless microsensor networks. In HICSS '00: Proceedings of the 33rd Hawaii International Conference on System Sciences-Volume 8, page 8020, Washington, DC, USA, 2000. IEEE Computer Society.

[7] M. Hollick, I. Martinovic, T. Krop, and I. Rimac. A survey on dependable routing in sensor networks, ad hoc networks, and cellular networks. In EUROMICRO '04: Proceedings of the 30th EUROMICRO Conference (EUROMICRO'04), pages 495-502, Washington, DC, USA, 2004. IEEE Computer Society.

[8] C. Intanagonwiwat, R. Govindan, and D. Estrin. Directed diffusion: a scalable and robust communication paradigm for sensor networks. In Mobile Computing and Networking, pages 56-67, 2000.

[9] A.-K. J.N. and K. A.E. Routing techniques in wireless sensor networks: a survey. Wireless Communications, IEEE, 11(6):6-28, 2004. 
[10] J. Kulik, W. R. Heinzelman, and H. Balakrishnan. Negotiation-based protocols for disseminating information in wireless sensor networks. Wireless Networks, 8(2-3):169-185, 2002.

[11] C.-J. Lin, P.-L. Chou, and C.-F. Chou. Hcdd: hierarchical cluster-based data dissemination in wireless sensor networks with mobile sink. In IWCMC '06: Proceedings of the 2006 international conference on Wireless communications and mobile computing, pages 1189-1194, New York, NY, USA, 2006. ACM Press.

[12] S. Lindsey and C. S. Raghavendra. Pegasis: Power-efficient gathering in sensor information systems, 2002.

[13] A. Nahapetian, S. Ghiasi, and M. Sarrafzadeh. Scheduling on heterogeneous resources with heterogeneous reconfiguration costs. In Proceedings of the fifteenth IASTED International Conference on Parallel and Distributed Computing and Systems, pages 916-921, Marina del Ray, CA, USA, 2003.

[14] M. Tubaishat and S. Madria. Sensor networks: an overview. IEEE Potentials, 22(2):20-23, 2003.

[15] W. Y. Yuan Li and J. Heidemann. Energy efficient network reconfiguration for mostly-off sensor networks. In 3rd Annual IEEE Communications Society on Sensor and Ad Hoc Communications and Networks, 2006. SECON '06, pages 527-535, 2006. 